\title{
An Iterative Method for the Solution of Eigenvalue Problems
}

\author{
By M. Godart
}

1. Introduction. Many boundary value problems of interest in mathematical physics can be finally reduced to the determination of the proper elements of a Sturm-Liouville equation. The most general form of these equations is:

$$
\frac{d}{d x}\left[p(x) \frac{d y(x)}{d x}\right]+q(x) y(x)+\lambda r(x) y(x)=0,
$$

with $a \leqq x \leqq b$, and the problem is to determine the particular values of the $\lambda$ parameter (eigenvalues) for which Eq. (1) possesses nonidentically zero solutions (eigenfunctions) obeying two boundary conditions of the type:

$$
\begin{aligned}
& A_{1} y(a)+A_{2} p(a) \frac{d y(a)}{d x}=0 \\
& B_{1} y(b)+B_{2} p(b) \frac{d y(b)}{d x}=0
\end{aligned}
$$

where the values of the constants $A_{1}, A_{2}$ and $B_{1}, B_{2}$ are not simultaneously zero.

Several methods have been proposed to determine the proper elements (i.e. eigenvalues and eigenfunctions) of Sturm-Liouville equations. Most of them have been reviewed by Kopal [1], but we shall examine one of them, the so called Rayleigh-Ritz method, in order to explain the main defect they have in common and to judge their general efficiency. This method was originally proposed by Ritz [2]. By transformations whose details will not be given here but which are described in many classical texts it leads to the solutions of equations of the form:

$$
\operatorname{det}\left\|D_{i k}-\lambda H_{i k}\right\|=0, \quad 1 \leqq i, k \leqq n
$$

where the $D_{i k}$ and $H_{i k}$ are the values of quadratic functionals for the $i$ th and $k$ th elements of a sequence of trial functions chosen once for all. Under rather general conditions, it can be shown that for indefinitely increasing values of $n$, the solutions of Eq. (3) decrease monotonically and converge to the eigenvalues of the originally stated problem. More precisely, if $\lambda_{m}{ }^{(n)}$ is the $m$ th solution of Eq. (3) when the solutions are ranged in increasing order, then the sequence of all the numbers $\lambda_{m}{ }^{(n)}$ with $n=m, m+1, \cdots$ is decreasing and converges to the $m$ th eigenvalue of the corresponding Sturm-Liouville equation when its eigenvalues are also ranged in increasing order. The big defect of this approximation method is its inability to furnish any estimate of the difference between one of the numbers $\lambda_{m}{ }^{(n)}$ and the corresponding eigenvalue $\lambda_{m}$. A theoretical convergence is not sufficient because the solution of Eq. (3) gets extremely complicated when $n$ increases. A rapid convergence is thus required but this can only be reasonably expected if we possess beforehand a rather precise knowledge of the general behaviour of the eigenfunctions. This is not usually the case. An even more serious defect of this method is that high values of $n$ are

Received October 27, 1965. 
necessary to obtain the higher order eigenvalues and to reduce a truncation-like error for the low order eigenvalues appreciably. When however such large values of $n$ are used, the determinantal Eq. (3) for $\lambda$ turns out to be of a correspondingly high degree and its solution may then entail such an accumulation of round-off errors as to prevent any further diminution of the total error affecting the computed eigenvalues. The necessity to compromise between these two sources of error severely restricts the accuracy obtainable. Similar restrictions are encountered when other previously developed methods are applied.

Thus, the methods in the current mathematical literature devoted to the problem of approximating the proper elements of a Sturm-Liouville problem are not very reliable. Specialists have, however, succeeded in improving those methods. Unfortunately their results are not very well known by the increasing number of scientists who are now able to program an electronic computer using one of the numerous machine oriented languages. For this reason, it seems interesting and useful to describe a new method which could be easily used by programmers who do not want to spend time with the theoretical numerical aspects of their problems. In what follows, the underlying principle of the method will be explained and it will be shown how it can be applied. A particularly simple case will then be treated in order to illustrate the efficiency of the new method. It will finally be shown that it can be extended to singular Sturm-Liouville equations.

2. The New Approximation Method. The method is based on the remarkable properties of a function introduced in a change of dependent variables that considerably simplifies the theoretical study of those equations. Continual reference will be made to references [4] and [5] where all details and proofs omitted here for sake of brevity can be found. It will be assumed that in the interval $a \leqq x \leqq b$, the function $p(x)$ is positive and possesses a first continuous derivative, that the function $r(x)$ is positive and continuous and that the function $q(x)$ is continuous. The new dependent variable $\rho(x)$ and $\theta(x)$ may be introduced by means of the defining equations:

$$
\begin{aligned}
y(x) & =\rho(x) \sin \theta(x), \\
p(x) \frac{d y(x)}{d x} & =\rho(x) \cos \theta(x) .
\end{aligned}
$$

According to Eq. (1) and boundary conditions (2, a) and (2, b), the function $\theta(x)$ obeys the differential equation:

$$
\frac{d \theta(x)}{d x}=\frac{1}{p(x)} \cos ^{2} \theta(x)+[q(x)+\lambda r(x)] \sin ^{2} \theta(x)
$$

and satisfies the boundary conditions:

$$
\begin{gathered}
A_{1} \sin \theta(a)+A_{2} \cos \theta(a)=0, \\
B_{1} \sin \theta(b)+B_{2} \cos \theta(b)=0,
\end{gathered}
$$

while the function $\rho(x)$ obeys the differential equation:

$$
\frac{d \rho(x)}{d x}=\rho(x) \sin \theta(x) \cos \theta(x)\left[\frac{1}{p(x)}-q(x)-\lambda r(x)\right]
$$


and satisfies the condition of never being zero in the interval $a \leqq x \leqq b$.

Let us now determine a priori permissible initial and final values for the function $\theta(x)$ by means of the auxiliary conditions:
$A_{1} \sin \alpha+A_{2} \cos \alpha=0$,
$0 \leqq \alpha<\pi$,
$B_{1} \sin \beta+B_{2} \cos \beta=0$,
$0<\beta \leqq \pi$.

Boundary conditions $(7, a)$ and $(7, b)$ can then be replaced by the equivalent conditions:

$$
\begin{aligned}
& \theta(a)=\alpha, \\
& \theta(b)=\beta+n \pi,
\end{aligned}
$$

where $n$ is any integer (positive, negative or null).

The solution $\theta(x, \lambda)$ of equation (6) satisfying an initial condition deduced from $(10, a)$ and $(9, a)$ possesses the following interesting properties.

As is shown in [4] and [5], $\theta(x, \lambda)$ is a monotonically increasing function of the argument $\lambda$ and satisfies the equalities:

$$
\begin{aligned}
& \lim _{\lambda \rightarrow-\infty} \theta(b, \lambda)=0, \\
& \lim _{\lambda \rightarrow+\infty} \theta(b, \lambda)=+\infty .
\end{aligned}
$$

Moreover, the function:

$$
\chi(x, \lambda)=\frac{\partial \theta(x, \lambda)}{\partial \lambda}
$$

is a solution of the differential equation:

$$
\frac{d \chi(x, \lambda)}{d x}=\chi(x, \lambda)\left[q(x)+\lambda r(x)-\frac{1}{p(x)}\right] \sin 2 \theta(x, \lambda)+r(x) \sin ^{2} \theta(x, \lambda)
$$

and it obviously satisfies the initial condition:

$$
\chi(a, \lambda)=0 .
$$

From this, we can deduce that $\chi(x, \lambda)$ is positive everywhere in the interval $a<$ $x \leqq b$. Equation (13) and initial condition (14) lead to the expression:

$$
\begin{aligned}
& \chi(x, \lambda)=\exp \int_{a}^{x}\left[q(\xi)+\lambda r(\xi)-\frac{1}{p(\xi)}\right] \sin 2 \theta(\xi, \lambda) d \xi \\
& \cdot \int_{a}^{x} r(\eta) \sin ^{2} \theta(\eta, \lambda) \exp \left\{-\int_{a}^{\eta}\left[q(\xi)+\lambda r(\xi)-\frac{1}{p(\xi)}\right] \sin 2 \theta(\xi, \lambda) d \xi\right\} d \eta
\end{aligned}
$$

Because of our hypothesis concerning the functions $p(x), q(x)$ and $r(x)$, this function is certainly nonnegative in the interval $a<x \leqq b$. Moreover, it can be zero at a point $x=c$ of this interval only if the function $\sin \theta(x, \lambda)$ is identically zero and thus constant in the interval $a<x \leqq c$. This cannot happen however because at all points where the function $\sin \theta(x, \lambda)$ vanishes, we have according to $(6)$ : 


$$
\frac{d \theta(x, \lambda)}{d x}=\frac{1}{p(x)}>0
$$

and also:

$$
\frac{d \sin \theta(x, \lambda)}{d x}= \pm \frac{1}{p(x)} \neq 0
$$

and this would contradict the previous deduction that $\sin \theta(x, \lambda)$ must be constant in the interval $a<x \leqq c$.

Now, the derivative $\chi(b, \lambda)$ of the function $\theta(b, \lambda)$ with respect to $\lambda$ is positive. When this is considered with the relations $(11, \mathrm{a})$ and $(11, \mathrm{~b})$ it may be clearly seen that the second boundary condition $(10, b)$ considered as an equation for $\lambda$ is solvable only for nonnegative values of the integer $n$ and that it then possesses one and only one solution. The calculation of the $(n+1)$ th eigenvalue $\lambda_{n}$ is thus equivalent to the solution of the equation:

$$
\beta+n \pi-\theta(b, \lambda)=0 .
$$

In the present case, the Newton-Raphson approximation method leads to the algorithm:

$$
\lambda_{n, k+1}=\lambda_{n, k}+\left[\beta+n \pi-\theta\left(b, \lambda_{n, k}\right)\right] \chi^{-1}\left(b, \lambda_{n, k}\right),
$$

where $\lambda_{n, k}$ denotes the $k$ th approximation to the eigenvalue $\lambda_{n}$. The application of the algorithm (19) does not present any difficulties, especially when an electronic computer is available for the numerical integration of Eqs. (6) and (13), or (6) and (15). Nothing can ensure the convergence of the successive estimates $\lambda_{n, k}$ to the corresponding eigenvalue $\lambda_{n}$, but obtaining a converging sequence of approximations is no longer a problem. In fact, it can be seen that the correction proposed by formula (19) for a known approximation is always in the right direction. In other words, this correction is positive (resp. negative, zero) if the chosen approximation is less than (resp. greater than, equal to) the sought eigenvalue. Then the only accident that must be avoided is to disturb or even to make the convergence impossible by obtaining successive approximations $\lambda_{n, i}, \lambda_{n, k}$ and $\lambda_{n, j}(i<k<j)$ as shown in the following diagram:

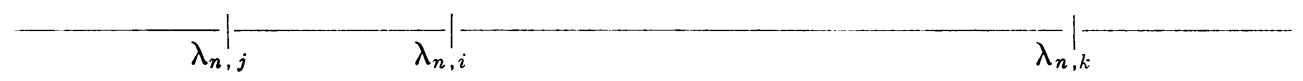

Whenever such a situation occurs, it is however possible to realise a sequence of successive approximations, which certainly converges to the sought eigenvalue $\lambda_{n}$, by modifying slightly the iteration process. As remarked just above, the sign of the correction proposed by formula (19) to a given approximation immediately shows if this value is a lower or a upper bound for the sought eigenvalue. At each step in the iteration process, a greatest lower bound $m$ and a least upper bound $M$ for $\lambda_{n}$ can thus be determined by using all preceding approximations. The last approximation obtained can then be accepted if it falls in the interior of the interval $m<\lambda<M$, but if it fell outside, it is necessary to replace it by some inner point of this interval, the middle point for example. There is then no difficulty in seeing that a sequence of successive approximations which is convergent to the sought eigenvalue is always obtained. 
TABLE I

\begin{tabular}{c|c|c|c|c}
\hline$n$ & $\lambda_{\text {comp }}$ & $\lambda_{\text {theor }}$ & $\epsilon \times 10^{6}$ & Iterations \\
\cline { 2 - 3 } 1 & 2.46739 & 2.467401 & 4 & 9 \\
2 & 22.2068 & 22.20661 & 8 & 1 \\
3 & 61.6863 & 61.68503 & 21 & 3 \\
4 & 120.907 & 120.9026 & 36 & 3 \\
& 199.869 & 199.8595 & 48 & 3 \\
\hline
\end{tabular}

\section{Applications.}

A. The efficiency of the proposed method for the approximation of the proper elements of Sturm-Liouville equations, will now be investigated on a particularly simple example.

Consider the differential equation:

$$
\frac{d^{2} y(x)}{d x^{2}}+\lambda y(x)=0
$$

and the boundary conditions:

$$
\begin{gathered}
y(0)=0, \\
\frac{d y(1)}{d x}=0 .
\end{gathered}
$$

Elementary calculations show that the proper elements of this problem are given by:

$$
\begin{aligned}
\lambda_{n} & =(2 n+1)^{2} \pi^{2} / 4, \\
y_{n}(x) & =\sin (2 n+1) \pi x / 2 .
\end{aligned}
$$

In Table I, we compare the eigenvalues as they have been determined by our method $\left(\lambda_{\text {comp }}\right)$ to their exact values $\left(\lambda_{\text {theor }}\right)$ for the first five values of the integer $n$. As an illustration, the relative errors and the required numbers of iterations are also given. It can thus be seen that the method provides good results in this case.

Practical problems are not so simple to handle and with many the mostimportant question is the determination of the relative errors. By considering formula (19) once more, it can be said from a naive point of view that if the method used to solve Eqs. (6) and (13), or (6) and (15) was perfectly exact, then the errors on the computed eigenvalues could be made arbitrarily small. This is however sufficient for claiming that the iteration process can be led in such a way that the error in the obtained results are introduced only when solving (6) and (13), or (6) and (15). This is seen if the maximum error $\epsilon$ for the quantity $\theta(b, \lambda)$ can be determined beforehand and if the iteration process is then stopped when the correction to a given approximation is much smaller than $\epsilon$.

As a last remark, it must be said that it is difficult to give any valuable indications on how much work is required in each iteration step: this depends too much on the adopted method for solving the involved differential equations. In the examples chosen here, as illustration, only a general method has been retained. This is due to the fact that the present differential equations can be solved very easily analytically, 
so that there is no natural limitation in the improvements that can be brought to the numerical solution of these equations.

B. The theory as developed to this point is not applicable to cases for which the function $p(x)$ can be zero at one or both of the extremities $x=a$ or $x=b$. The method proposed is however applicable to these cases, after being slightly modified For sake of brevity, the theoretical aspects will be omitted and only intuitive arguments will be used. These can, however, be established rigorously. Two examples will be treated in order to indicate the suggested extension.

1. Consider the differential equation:

$$
\frac{d}{d x}\left[x \frac{d y(x)}{d x}\right]+\lambda x y(x)=0
$$

and the boundary conditions:

$$
\begin{gathered}
|y(0)|<+\infty, \\
y(1)=0 .
\end{gathered}
$$

It can easily be seen that the proper elements of this Sturm-Liouville problem are given by:

$$
\begin{aligned}
\lambda_{n} & =j_{n}{ }^{2}, \\
y_{n}(x) & =x^{1 / 2} J_{0}\left(j_{n} x\right),
\end{aligned}
$$

where $J_{0}$ is the Bessel function of order 0 and where $j_{n}$ is its $(n+1)$ th positive zero.

Equation (6) shows that the singularity at $x=0$ can be avoided if we take:

$$
\alpha=\pi / 2 \text {. }
$$

By choosing $\beta$ as explained in the second paragraph, the approximation method may be applied. The calculations have been performed and the obtained results are given in Table II in the same form as used for Table I. Once more, the method has given reliable results.

2. Consider finally the differential equation:

$$
\frac{d}{d x}\left[\left(1-x^{2}\right) \frac{d y(x)}{d x}\right]+\lambda y(x)=0
$$

and the boundary conditions:

$$
\begin{aligned}
& |y(-1)|<+\infty, \\
& |y(+1)|<+\infty,
\end{aligned}
$$

It is well known that the proper elements of this problem are given by:

$$
\begin{aligned}
\lambda_{n} & =n(n+1), \\
y_{n}(x) & =P_{n}(x),
\end{aligned}
$$

where $P_{n}(x)$ is the Legendre polynomial of order $n$. Equation (6) shows that the singularity at $x=-1$ can be avoided if as before:

$$
\alpha=\pi / 2 .
$$


TABLE II

\begin{tabular}{c|c|c|c|c}
\hline$n$ & $\lambda_{\text {comp }}$ & $\lambda_{\text {theor }}$ & $\epsilon \times 10^{6}$ & Iterations \\
\cline { 2 - 3 } 0 & 5.78307 & 5.7831862 & 20 & 10 \\
1 & 30.47111 & 30.471262 & 5 & 10 \\
2 & 74.88676 & 74.887006 & 3 & 11 \\
3 & 139.0414 & 139.04027 & 8 & 11 \\
4 & 222.9352 & 222.93231 & 13 & 9 \\
\hline
\end{tabular}

TABLE III

\begin{tabular}{c|c|c|c|c}
\hline$n$ & $\lambda_{\text {comp }}$ & $\lambda_{\text {theor }}$ & $\epsilon \times 10^{6}$ & Iterations \\
\cline { 1 - 3 } 0 & 0 & 0 & - & 1 \\
1 & 1.99997 & 2 & 15 & 8 \\
2 & 5.99984 & 6 & 27 & 10 \\
3 & 11.9996 & 12 & 33 & 9 \\
4 & 19.9991 & 20 & 45 & 13 \\
\hline
\end{tabular}

The method however is no longer applicable in its original form because of the second singularity at $x=+1$, yet its basic idea may be employed. For this, introduce some intermediate point, say $x=0$. For an arbitrary value of $\lambda$, the equations (6) and (13) or (6) and (15) can be solved in the interval $-1 \leqq x \leqq 0$ taking the initial conditions $(14),(10, a)$ and $(26, a)$ into account. The values at $x=0$ of the functions $\theta(x, \lambda)$ and $\chi(x, \lambda)$ just obtained are then denoted by $\theta_{L}(\lambda)$ and $\chi_{L}(\lambda)$.

In exactly the same manner, the singularity at $x=1$ can be avoided by choosing:

$$
\beta=\pi / 2 \text {. }
$$

Taking into account the initial conditions $(14),(10, \mathrm{~b})$ and $(26, \mathrm{~b})$, the equations (6) and (13) or (6) and (15) can be solved in the interval $0 \leqq x \leqq 1$. The values at $x=0$ of the new functions $\theta(x, \lambda)$ and $\chi(x, \lambda)$ that have been obtained are then denoted by $\theta_{R}(\lambda)$ and $\chi_{R}(\lambda)$.

It can be shown that the derivative $\chi_{L}(\lambda)$ of the function $\theta_{L}(\lambda)$ with respect to $\lambda$ is always positive and that the derivative $\chi_{R}(\lambda)$ of the function $\theta_{R}(\lambda)$ with respect to $\lambda$ is always negative. Moreover, the eigenvalues are the solutions of the equation:

$$
\theta_{L}(\lambda)-\theta_{R}(\lambda)=0 .
$$

Equation (19) can then be replaced by the new algorithm:

$$
\lambda_{n, k+1}=\lambda_{n, k}-\left[\theta_{R}\left(\lambda_{n, k}\right)-\theta_{L}\left(\lambda_{n, k}\right)\right] /\left[\chi_{R}\left(\lambda_{n, k}\right)-\chi_{L}\left(\lambda_{n, k}\right)\right] .
$$

This new form of the approximation method has been applied to the determination of the eigenvalues of the Sturm-Liouville problem defined by the relations (27), $(28, \mathrm{a})$ and $(28, \mathrm{~b})$. The results obtained in this case are summarized in Table III. Once more, the comparison shows that the method has provided excellent results.

4. Conclusion. A new method of successive approximations has been proposed in order to solve eigenvalue and eigenfunction problems associated with SturmLiouville equations. The examples treated show that the convergence is reasonably 
rapid and that the proper elements can be determined with an actual accuracy which is only limited by the errors inherent to the numerical resolution of Eqs. (6), (13) and (15). It is thus highly recommended to replace numerical determinations by analytic expressions whenever this is possible. The number of iterations of the approximation method is highly dependent on the first estimates chosen for the $\lambda_{n}$. These values must then be determined as accurately as possible either by comparison methods, by an asymptotic expression, or by any other means. In all cases, some theoretical study is always helpful for the numerical solution of a Sturm-Liouville problem.

5. Acknowledgment. The author would like to express his thanks to the I.R.M. (Belgian Royal Meteorological Institute) for its assistance in making available its IBM 7040 computer. It was used for the solution of the examples given above and for a practical case arising in the study of a diffusion problem.

Institut d'Aeronomie Spatiale de Belgique,

3 , Avenue Circulaire,

Bruxelles 18, Belgique

1. Z. Kopal, Numerical Analysis, Wiley, New York, 1955. MR 17, 1007.

2. W. RITz, "Über eine neue Methode zur Lösung gewisser Variationsprobleme der mathematischen Physik," J. Reine Angew. Math., v. 135, 1909, pp. 1-61.

3. S. G. Mikhun, Integral Equations and Their Applications to Certain Problems in Mechanics, Pergamon, London, 1964, MR 29 \#1508.

4. H. PRÜFER, "Neue Herleitung der Sturm-Louvilleschen Reihenentwicklung stetiger Funktionen," Math. Ann., v. 95, 1926, pp. 499-518.

5. E. Kamke, Differentialgleichungen reeller Funktionen, Akademische Verlagsgesellschaft, Leipzig, 1930. 